\title{
Hypersensitivity to Intravenous Ondansetron
}

\author{
Ibel Chiramel Fredy ${ }^{1 *}$ and Nimisha Ganesh ${ }^{2}$ \\ ${ }^{1}$ Trinity College, Doctor of Pharmacy, PES College of Pharmacy, India \\ ${ }^{2}$ Doctor of Pharmacy, PES College of Pharmacy, India
}

Received: September 21, 2017; Published: October 02, 2017

*Corresponding author: Ibel Chiramel Fredy, Msc Neuroscience (Trinity College Dublin), Doctor of Pharmacy (Pharm D) PES College of Pharmacy, Bangalore, Karnataka, India, Email: ibelcfredy@gmail.com

\begin{abstract}
A 33 year old male who was recovering after cholecystectomy presented a sudden onset of breathing difficulty and rashes all over the body with inj. Andtron (Ondansetron -4mg). The condition progressed to cardio pulmonary arrest, which was fortunately resuscitated successfully. Ondansetron is a highly potent antiemetic drug that is effective in preventing postoperative nausea and vomiting with very low risk of adverse reactions. We are reporting a reaction that is probably due to an allergy to serotonin blockers (Ondansetron, Granisetron). The patient recovered on discontinuing the drug and with appropriate treatment.
\end{abstract}

Abbreviations : PONV: Postoperative Nausea and Vomiting

\section{Introduction}

Post operative nausea and vomiting are unpleasant complications of surgery and anesthesia. Antiemetic is frequently used in their management. Ondansetron is highly potent selective serotonin 5-HT3 receptor antagonist. Considered safe and effective in preventing chemotherapy- and-radiation-induced nausea and vomiting as well as vomiting in postoperative patients. When used in these clinical circumstances, there is very low risk of significant adverse effects, favorable safety profile and absence of drowsiness as side effect. $[1,2]$. However, unlikely but serious side effects may occur: stomach pain, muscle spasm, vision changes, chest pain, slow / fast / irregular heartbeat, dizziness, fainting. A very serious allergic reaction to this drug is rare but, if it occurs stop taking this drug and seek immediate medical attention. Symptoms of a serious allergic reaction include: rash, itching or swelling, severe dizziness, trouble breathing $[3,4]$.

\section{Case report}

A 33 year old male underwent a Cholecystectomy procedure for abdominal adhesion. The abdominal adhesion in this case is probably due to a previous surgery the patient underwent 3 months' prior after an intestinal perforation by steel rod in an accident. After the successful procedure, the patient was moved to ICU, the Ryle's tube was put up for aspiration of stomach contents. After one week, the Ryle's tube was removed as the stomach aspirate was found to be normal $(300 \mathrm{ml})$ and the patient presented no distention and he has passed flatus. He was prescribed with inj. Ondansetron (4mg) given half an hour before starting oral fluids after auscultating the stomach peristalsis. On receiving Ondansetron, the patient became distressed, with rashes all over the body (back, face and arms) and Cardiopulmonary arrest. The patient was successfully revived by timely resuscitation and inj. Avil (4mg) and Hydrocort (40mg). The reaction abated within a few minutes and he was asymptomatic at 72 hours of follow up. He had no history of ondansetron exposure, or drug or food allergies.

\section{Discussion}

Postoperative nausea and vomiting (PONV) are the most common and distressing complications after surgery and anesthesia, and may lead to serious postoperative complications. The average incidence of PONV has been reported to be $25 \%$, but can increase up to $80 \%$ in patients with several risk factors, such as sex, non-smoking, prior history of motion sickness or PONV, and the use of post-operative opioids [5]. Ondansetron, a highly selective 5-HT3 receptor antagonist, is effective in the prevention and treatment of PONV. While its mechanism has not been fully characterized, it is found to prevent emesis by antagonizing the action of 5-hydroxy tryptamine (5-HT) at 5-HT3 receptors n vagal afferent neurons that innervate the gastrointestinal tract and 5-HT3 receptors in central vomiting system $[4,5]$. Ondansetron is a welltolerated drug. Constipation, diarrhea, and headache are the most commonly reported side effects. Unlikely but serious side effects such as stomach pain, muscle spasm, vision changes (temporary loss of sight blurred vision) may occur.

5-HT3 receptor antagonists (such as Ondansetron, Tropisetron, Granisetron, and Palonosetron), though associated with a wide 
safety margin shows unusual reports of life threatening adverse events such as generalized tonic clonic seizures, hypotension, chest pain, dystonia and anaphylaxis. Hypersensitivity reactions characterized by rashes, swelling, shortness of breath and cardiopulmonary arrest have been reported in patients who have exhibited hypersensitivity to other selective 5-HT3 receptor antagonists. However, some authors suggest that the anaphylaxis may be a class effect while others think it may be drug specific [6].

\section{Conclusion}

On the Naranjo's causality assessment scale, the adverse event was 6 indicating a "probable" reaction to Ondansetron. In this case the reaction could be either anaphylaxis or Anaphylactoid, but latter seems more likely given the history of absence of prior sensitization [6,7]. Other components of the drug, such as solvent, also need to be considered as a cause of the reaction. Considering all of the existing evidence, we emphasize the need to be cautious when administering the ondansetron and to be aware of the unusual adverse occurrences.

\section{References}

1. Gan TJ (2006) Risk factors for post operative nausea and vomiting database research: limitations and opportunities. AnesthAnalg 102(6): 1884-1898.

2. Habib AS, Gan TJ (2010) Post operative nausea and vomiting database research: limitations and research opportunities. AnesthAnalg 110(2): 412-414.

3. Eduardo D (1998) Ondansetron in the prophylaxis of postoperative vomiting: a meta-analysis. Journal of clinical anaesthasia 10(3): 211221.

4. Tripathi KD (2008) Essentials of medical pharmacology. $6^{\text {th }}$ ed: Jaypee Brothers Medical publishers Ltd, New Delhi, India, pp. 171-175.

5. HS Smith, EJ Smith (2012) Postoperative nausea and vomiting. APM $1(2)$.

6. Chen M, Tanner A, Gallo Torres H (1993) Anaphylactoid Anaphylactic reactions associated with ondansetron. Ann Intern Med 119(8): 862.

7. Beverley Greenwood Van Meerveld (2017) Handbook of experimental pharmacology. $1^{\text {st }}$ edition, Canada, North America, pp. 239.

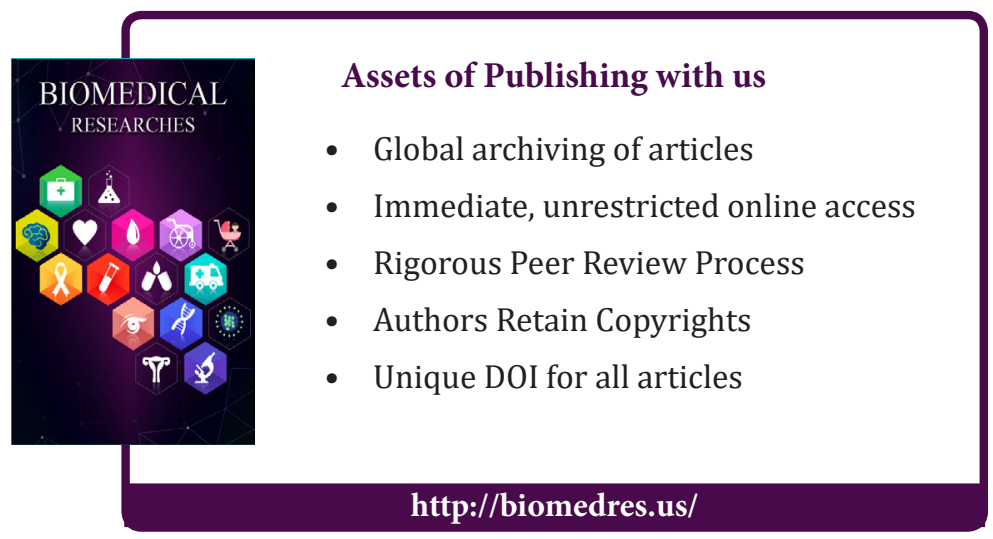

